\title{
Respiratory failure with diffuse bronchiectases and cryoglobulinaemia
}

\author{
N. Girard ${ }^{*,+}$, A. Vasiljevic ${ }^{\#,+}$, V. Cottin*, L. Falchero", \\ , D. Meyronet ${ }^{\#}$, F. Thivolet-Bejui ${ }^{\#}$ and J-F. Cordier*
}

\section{CASE REPORT}

A 48-yr-old nonsmoking female presented with a 5-yr history of recurrent bronchitis with progressive exercise dyspnoea. She had been diagnosed with a small monoclonal gammopathy of undermined significance (MGUS) 6 yrs previously. Inspiratory vital capacity was $4.7 \mathrm{~L}(125 \%$ predicted) and forced expiratory volume in one second/forced vital capacity (FVC) ratio was $79 \%$. Carbon monoxide transfer factor was markedly impaired (35\% pred), and arterial oxygen tension $\left(\mathrm{Pa}_{1} \mathrm{O}_{2}\right)$ decreased from 9.2 to $6.0 \mathrm{kPa}$ at exercise $(40 \mathrm{~W}, 10 \mathrm{~min})$. A thoracic computed tomography (CT) scan was performed (fig. 1). Fibreoptic bronchoscopy found inflammation of the bronchial mucosa with candle stain-like deposits on the trachea and large bronchi, without any purulent secretion within the respiratory tract (fig. 2). The histopathological aspect of bronchial biopsies is shown in figure 3. Blood tests disclosed type I cryoglobulinaemia composed of a $32 \mathrm{~g} \cdot \mathrm{L}^{-1}$ monoclonal immunoglobulin (Ig)Mк serum component (at $37^{\circ} \mathrm{C}$ ). Blood cell count was normal. Bone marrow examination showed malignant lymphoplasmacytic cells representing $30 \%$ of total cells, with a monotypic surface expression of IgMк. The C-reactive protein level was $21 \mathrm{mg} \cdot \mathrm{L}^{-1}$. Extensive renal, cardiac and hepatic evaluations were normal. As severe hypoxaemia and reduction of carbon monoxide transfer factor were unexplained, the patient underwent videothoracoscopic lung biopsies.

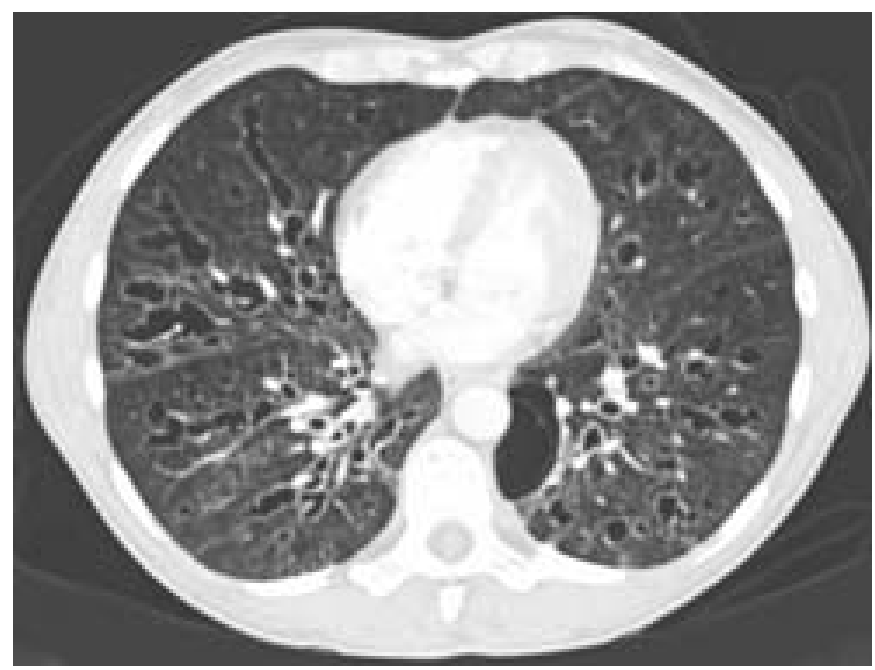

FIGURE 1. Thoracic computed tomography scan.

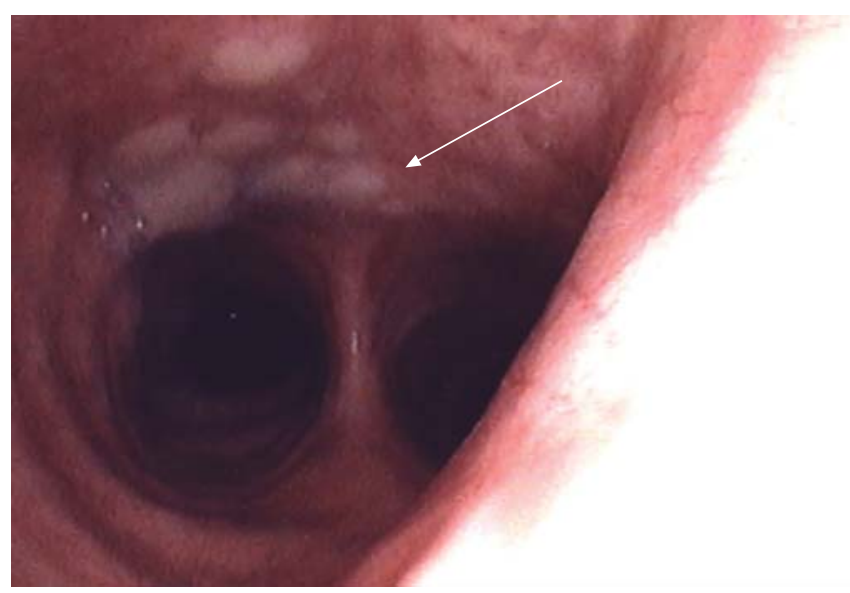

FIGURE 2. Fibreoptic bronchoscopy. Arrow shows candle stain-like deposits.

*Reference Center for Rare Lung Diseases, Dept of Respiratory Medicine, and \#Pathology Center, Louis Pradel Hospital, Hospices Civils de Lyon, Lyon I University, Lyon and "Dept of Respiratory Medicine, Villefranche-sur-Saône Hospital, Villefranche-sur-Saône, France, ${ }^{+B}$ Both authors contributed equally to this work. 

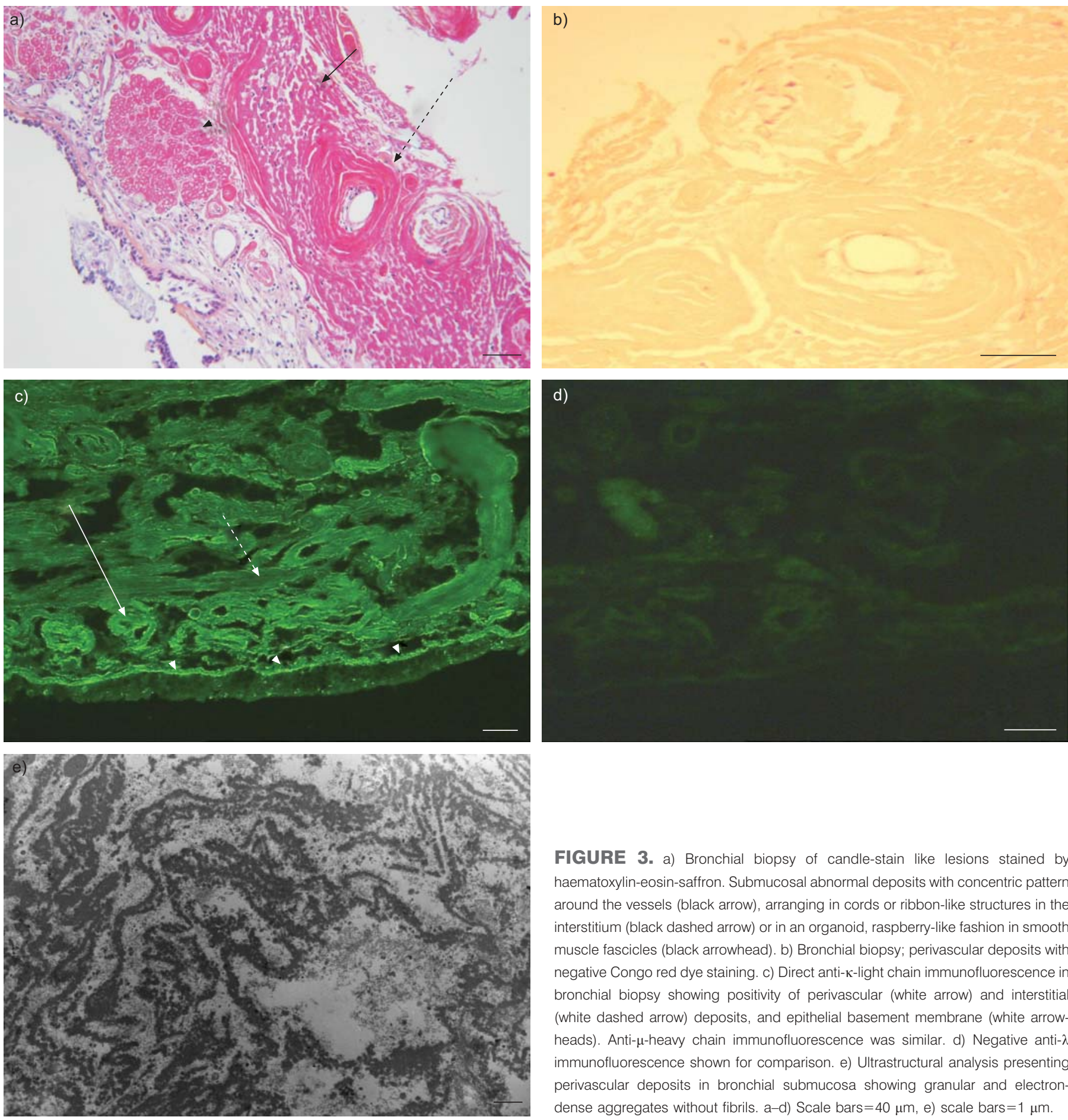

FIGURE 3. a) Bronchial biopsy of candle-stain like lesions stained by haematoxylin-eosin-saffron. Submucosal abnormal deposits with concentric pattern around the vessels (black arrow), arranging in cords or ribbon-like structures in the interstitium (black dashed arrow) or in an organoid, raspberry-like fashion in smooth muscle fascicles (black arrowhead). b) Bronchial biopsy; perivascular deposits with negative Congo red dye staining. c) Direct anti-k-light chain immunofluorescence in bronchial biopsy showing positivity of perivascular (white arrow) and interstitia (white dashed arrow) deposits, and epithelial basement membrane (white arrowheads). Anti- $\mu$-heavy chain immunofluorescence was similar. d) Negative anti- $\lambda$ immunofluorescence shown for comparison. e) Ultrastructural analysis presenting perivascular deposits in bronchial submucosa showing granular and electrondense aggregates without fibrils. a-d) Scale bars $=40 \mu \mathrm{m}$, e) scale bars $=1 \mu \mathrm{m}$. BEFORE TURNING THE PAGE, INTERPRET THE CLINICAL, RADIOLOGICAL AND
PATHOLOGICAL DATA, AND SUGGEST A DIAGNOSIS. 


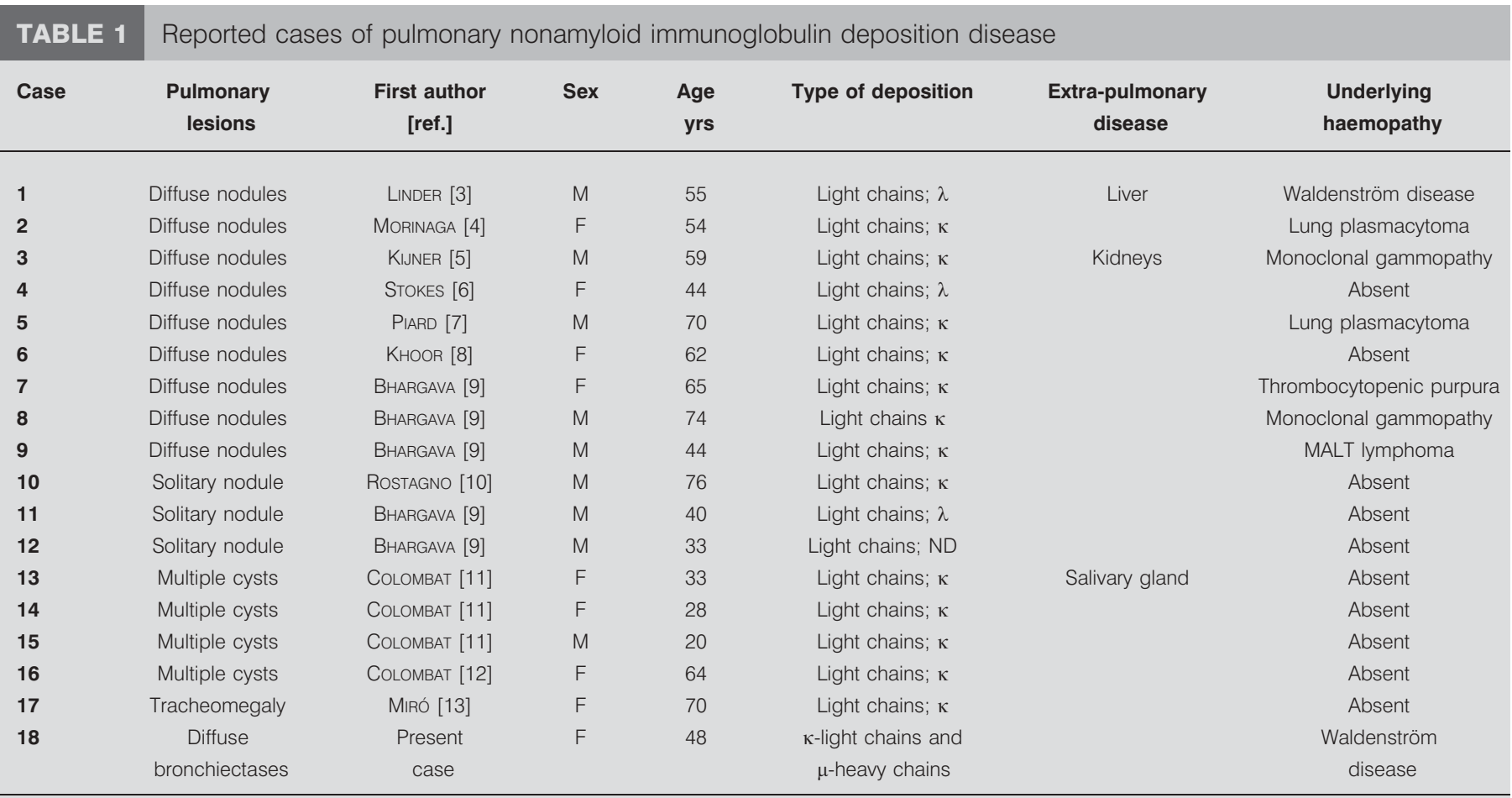

M: male; F: female; MALT: mucosa-associated lymphoid tissue; ND: not determined.

\section{INTERPRETATION}

\section{CT scan}

CT features consisted of diffuse thin-walled moniliform bronchiectases, associated with a sub-pleural cystic lesion in the left lower lobe. No nodules or diffuse interstitial pneumonia were present.

\section{Lung function}

Lung function testing did not demonstrate any ventilatory defect, contrasting with a marked decrease of gas exchange and severe exercise hypoxaemia. This pattern, together with decreased carbon monoxide transfer factor, suggests an alteration in the alveolar-capillary membrane.

\section{Blood tests}

The high blood level of IgM monoclonal component $\left(>30 \mathrm{~g} \cdot \mathrm{L}^{-1}\right)$ associated with the bone marrow infiltration by monotypic lymphoplasmacytic cells $(>10 \%)$ allowed the diagnosis of Waldenström macroglobulinaemia [1]. The previous diagnosis of MGUS was initially performed because previous measurements of monoclonal IgM level had been notably underestimated, due to its cryoglobulinic property requiring the assay to be performed at $37^{\circ} \mathrm{C}$ in order to ensure accurate determination. Nonetheless, in a patient of only $44 \mathrm{yrs}$ of age, close follow-up of MGUS is warranted.

\section{Lung pathology}

Pathological examination of the bronchial and pulmonary biopsies displayed a diffuse deposition of an acellular eosinophilic material settling in the bronchi, bronchioles, vessels and alveolar walls. Deposits predominated in bronchi (fig. 3a) and bronchioles with peculiar affinity for epithelial basement membranes. Pulmonary gas exchange zones were involved, with slight deposits interposed between the alveolar lumen and the capillary wall. Staining with Congo red dye was negative (fig. $3 b$ ) and demonstrated no apple-green birefringence on polarised light microscopy, ruling out the diagnosis of amyloidosis. Immunofluorescence analysis demonstrated that these deposits consisted of $\kappa$-light chains and $\mu$-heavy chains (fig. 3c). Ultrastructural examination showed granular and electron-dense aggregates, but no amyloid fibrils (fig. 3d). The histopathological data were diagnostic of nonamyloidotic monoclonal immunoglobulin deposition disease (NAMIDD).

Diagnosis: Bronchopulmonary NAMIDD, secondary to Waldenström macroglobulinaemia with IgM-к cryoglobulin

\section{CLINICAL COURSE}

The patient was treated with corticosteroids, without clinical or biological improvement. She then received eight courses of rituximab, leading to a reduction of the serum monoclonal IgM- $\kappa$ component to $10.4 \mathrm{~g} \cdot \mathrm{L}^{-1}$ and transient improvement in exercise dyspnoea. However, lung function and arterial blood gas values deteriorated: FVC decreased from 4.7 to $4.0 \mathrm{~L}$; carbon monoxide transfer factor decreased from 35 to $14 \%$ pred; and $\mathrm{Pa}_{1} \mathrm{O}_{2}$ at rest decreased from 9.2 to $7 \mathrm{kPa}$. CT scan features were unchanged. Permanent oxygen therapy was started. Second-line treatment with fludarabine and rituximab (six courses), followed by maintenance with rituximab, led to a dramatic and sustained decrease of the monoclonal component $\left(0.9 \mathrm{~g} \cdot \mathrm{L}^{-1}\right)$. The clinical respiratory condition and lung function tests are currently stabilised, 3 yrs after the diagnosis. 


\section{DISCUSSION}

Monoclonal Ig deposition diseases are defined as visceral and soft tissue immunoglobulinic deposition, often resulting in organ failure [2]. They fall into two main categories, depending on the physical and spatial conformation of the deposits. Amyloidosis results from the deposition of Ig material (mostly of the $\lambda$-light chain isotype as in systemic amyloidosis of immunoglobulin origin (AL) amyloidosis), with reduced folding stability. Deposits organise into $\beta$-pleated sheet and further assemble into fibrillar bundles responsible for staining with Congo red dye and green birefringence under polarised light microscopy [2].

In contrast, NAMIDD presents with Congo red dye negative non-birefringent deposits, mostly consisting of $\kappa$-light chains (such as in the so-called Randall disease of the kidney) or, less frequently, of heavy chains, or of mixed light and heavy chains (table 1) [2]. Systemic NAMIDD is much rarer than amyloidosis and is more often associated with multiple myeloma than AL amyloidosis [2].

Like AL amyloidosis, NAMIDD predominantly involves the kidney and the heart, but lung involvement is exceptional, with only 17 cases reported [3-13]. Pulmonary NAMIDD may present as multiple parenchymal nodules (nine cases) [3-9] or as a single mass (three cases) $[9,10]$. A unique presentation of lymphangioleiomyomatosis-like polycystic lung disease has also been described recently (four cases) [11, 12]. In 14 patients, the deposition was apparently limited to the lung without systemic involvement. Association with haematological malignancy was found in only seven cases (six with multiple nodules, and the present case; table 1).

A hitherto undescribed clinical and immunohistopathological presentation of pulmonary NAMIDD characterised by the mixed deposition of light and heavy chains associated with type I IgM cryoglobulin and by diffuse involvement of the lower respiratory tract from the trachea to the alveolarcapillary membrane is reported herein. Biochemical analysis of the deposits was not performed, although they might presumably be composed of both light and heavy chains (or fragments of these), of IgM subunits or of intact IgM (an unlikely hypothesis considering the high molecular weight of $\operatorname{IgM}$ that precludes its extravasation in tissues). Contrary to amyloidosis, NAMIDD rarely affects the tracheobronchial tree, as only one other case has been reported, presenting with tracheobronchomegaly [13]. A macroscopic candle stain-like deposit on the bronchial mucosa has not been described previously. Deposition may be found at histopathology throughout the bronchopulmonary structures in the absence of macroscopic tracheobronchial abnormality and NAMIDD may be diagnosed by bronchial biopsies [12]. In the present patient, the deposition along the airways is likely to have caused the development of diffuse bronchiectases. Bronchiolar involvement was illustrated by the "raspberry-like pattern", a striking pathological feature suggestive of NAMIDD (fig. 3), which corresponds to interstitial deposits surrounding every atrophic myocyte of smooth muscle fascicles in the bronchiole.

The severe impairment of gas exchanges leading to respiratory failure in the present patient is another novel feature of NAMIDD and may be explained by the Ig deposition under the basement membrane between the alveolar and the capillary cells, as demonstrated by immunohistochemistry, which is also reported in cystic NAMIDD [11]. The severity of respiratory impairment in amyloidosis also largely depends upon the degree to which the gas exchange zone is affected [14].

As in amyloidosis, the treatment of pulmonary nonamyloidotic monoclonal immunoglobulin deposition disease aims at controlling the secretion of the serum monoclonal component and further progression of the deposits, and includes symptomatic management of the pulmonary manifestations, especially respiratory failure. Treatment of the underlying haematological disease when present allows the regression of the monoclonal component in most cases [15] but is poorly effective on existing deposits. In a similar manner to its renal counterpart, Randall disease, pulmonary nonamyloidotic monoclonal immunoglobulin deposition disease may benefit from lung transplantation in case of severe respiratory failure in the absence of overt and uncontrolled underlying haematological disorder [11].

\section{ACKNOWLEDGEMENTS}

The authors would like to thank F. Dijoud and D. Gindre (both Pathology Center, Louis Pradel Hospital, Lyon, France) for their participation in the pathological analysis, and D. Espinouse and G. Salles (both Dept of Haematology, Centre Hospitalier Lyon Sud, Hospices Civils de Lyon, Lyon, France) for participation in the clinical care of the patient.

\section{REFERENCES}

1 Bladé J. Clinical practice. Monoclonal gammopathy of undetermined significance. N Engl J Med 2006; 355: 2765-2770.

2 Buxbaum J, Gallo G. Nonamyloidotic monoclonal immunoglobulin deposition disease. Light-chain, heavy-chain, and light- and heavy-chain deposition diseases. Hematol Oncol Clin North Am 1999; 13: 1235-1248.

3 Linder J, Croker BP, Vollmer RT, Shelburne J. Systemic $\kappa$ light-chain deposition. An ultrastructural and immunohistochemical study. Am J Surg Pathol 1983; 7: 85-93.

4 Morinaga S, Watanabe H, Gemma A, et al. Plasmacytoma of the lung associated with nodular deposits of immunoglobulin. Am J Surg Pathol 1987; 11: 989-995.

5 Kijner $\mathrm{CH}$, Yousem SA. Systemic light chain deposition disease presenting as multiple pulmonary nodules. A case report and review of the literature. Am J Surg Pathol 1988; 12: 405-413.

6 Stokes MB, Jagirdar J, Burchstin O, Kornacki S, Kumar A, Gallo G. Nodular pulmonary immunoglobulin light chain deposits with coexistent amyloid and nonamyloid features in an HIV-infected patient. Mod Pathol 1997; 10: 1059-1065.

7 Piard F, Yaziji N, Jarry O, et al. Solitary plasmacytoma of the lung with light chain extracellular deposits: a case report and review of the literature. Histopathology 1998; 32: 356-361.

8 Khoor A, Myers JL, Tazelaar HD, Kurtin PJ. Amyloid-like pulmonary nodules, including localized light-chain deposition: clinicopathologic analysis of three cases. Am J Clin Pathol 2004; 121: 200-204.

9 Bhargava P, Rushin JM, Rusnock EJ, et al. Pulmonary light chain deposition disease: report of five cases and review of the literature. Am J Surg Pathol 2007; 31: 267-276. 
10 Rostagno A, Frizzera G, Ylagan L, Kumar A, Ghiso J, Gallo G. Tumoral non-amyloidotic monoclonal immunoglobulin light chain deposits ("aggregoma"): presenting feature of B-cell dyscrasia in three cases with immunohistochemical and biochemical analyses. Br J Haematol 2002; 119: 62-69.

11 Colombat M, Stern M, Groussard O, et al. Pulmonary cystic disorder related to light chain deposition disease. Am J Respir Crit Care Med 2006; 173: 777-780.

12 Colombat M, Gounant V, Mal H, Callard P, Milleron B. Light chain deposition disease involving the airways: diagnosis by fibreoptic bronchoscopy. Eur Respir J 2007; 29: 1057-1060.

13 Miró O, Fernández-Solá J, Gómez-Angelats E, Andreu MV, Solé M. [Tracheobronchomegaly associated with light chain deposition disease]. Arch Bronconeumol 1994; 30: 508-510.

14 Cordier JF, Loire R, Brune J. Amyloidosis of the lower respiratory tract. Clinical and pathologic features in a series of 21 patients. Chest 1986; 90: 827-831.

15 Royer B, Arnulf B, Martinez F, et al. High dose chemotherapy in light chain or light and heavy chain deposition disease. Kidney Int 2004; 65: 642-648. 